\title{
Review of: "A glycosylphosphatidylinositol-anchored $\alpha$-amylase encoded by amyD contributes to a decrease in the molecular mass of cell wall $\alpha-1,3-$ glucan in Aspergillus nidulans"
}

\author{
Xiaoxiao $\mathrm{He}^{1}$ \\ 1 Northeast Normal University
}

Potential competing interests: The author(s) declared that no potential competing interests exist.

In this manuscript, Ken and colleagues studied the function of AmD by constructing AmyD KO or overexpression strains in different A. nidulans parental strains. Their results showed AmyD mainly decrease the molecular mass of alpha-glucan, and it showed biased effect on alpha-glucan that produced by different alpha-glucan synthases. Based on these results, they proposed a interesting mechanism of AmyD to affect alpha-glucan synthesis and modification. It may be important to explaint the difference of AsgA- or AgsBderived alpha-glucan. However, the current data could not fully support this hypothesis. Some extra results are further required.

Major point:

1 According to previous reports (He et al., 2014 and 2017), AmyD repressed the accumulation of alphaglucan in A. nidulans cell wall. Both deletion or over-expression of AmyD had direct impacts on alphaglucan content in cell wall. Data in this manuscript showed that alpha-glucan was only affected in AmyD over-expression strains. It is unclear why AmyD deletion had no impact on alpha-glucan accumulation in cell wall.

2 Quantificaiton of glucose in AS2 fraction of $\triangle$ amyD strain presented both in figure 3A and figure 7A. There is a quite big difference for these two results.

3 The error bar for agsA-OE; amyD-OE strain in figure 3B was big compared with other quantification data. This would greatly affect the outcomes of statistical analysis. Authors should re-test this experiment to draw a better conclusion.

4 Line 426-434, authors hypothesized that AmyD might hydrolyze the primer of alpha-glucan and it may compete with the alpha-glucan synthase. This could be the mechanism of AmyD to reduce the Molecular mass of alpha-glucan. However, data in van de Kaaij et al., 2007 showed AgtA (the homolog of AmyD) mainly function as a glucanotransferase, which had very limited starch hydrolysis ability. Could AmyD has a function to modify the alpha-glucan in cell wall, as it suggested in line 435-445.

5 Is that possible the alpha-glucan in amyD-OE or agsB-OE; amyD-OE cross-linked with other cell wall component and making it alkaline insoluble. If so, it would be able to find the glucose concentration increased in other cell wall fractions. 
6 An enzymatic characterization would be needed to answer how exactly AmyD participate in alpha-glucan synthesis or modification.

Minor points:

1 The manuscript is particular long with many redundant paragraphs or sentences. For example. the third paragraph of Introduction, which summarized the function of Ags protein in all Apspergillus species. However, most of such information was not relevant to the current study.

2 There are mistakes in genotype of Table 1 for the last two strains.

3 It was not clearly described in the manuscript that the agsA-OE strain was constructed in $\triangle$ agsB background. And the agsB-OE strain was constructed in $\triangle$ agsA background. Such information is necessary to understand the data in this manuscript. 